Solid State Communications, Vol. 14,pp. 1217-1221, 1974. Pergamon Press. Printed in Great Britain

\title{
PLASMA OSCILLATIONS IN SPHERES AND THE MAGNETORESISTANCE OF POTASSIUM
}

\author{
S.A. Werner \\ Physics Dept., Scientific Research Staff, Ford Motor Co., Dearborn, Mich., U.S.A. \\ Department of Nuclear Engineering, University of Michigan, Ann Arbor, Mich., U.S.A. \\ T.K. Hunt \\ Physics Dept., Scientific Research Staff, Ford Motor Co., Dearborn, Mich., U.S.A. \\ and \\ G.W. Ford \\ Physics Department, University of Michigan, Ann Arbor, Michigan, U.S.A.
}

(Received 5 February 1974 by M. Cardona)

\begin{abstract}
We have observed in potassium the spectrum of the longitudinal helicon oscillations. We have used these oscillations to measure the magnetoresistance and Hall coefficient. In addition, a low frequency method for the measurement of magnetoresistance in metals is demonstrated with experiments on single crystal potassium spheres. The magnetoresistance saturates, but its magnitude depends upon sample history.
\end{abstract}

IN THIS paper we report the first observation of the longitudinal helicon spectrum ${ }^{1}$ in metal spheres, demonstrate a new method for the accurate measurement of the magnetoresistance of metals, and present low temperature results for potassium. In magnetoresistance measurements on the alkali metals, for which problems associated with sample fabrication and with making appropriate electrical contacts are severe, much effort has been spent on inductive techniques. ${ }^{2}$ Unfortunately, until recently no calculation of sufficient accuracy for examination of the details of the conductivity has been available for a finite sample geometry at arbitrary frequency and magnetic field. A recent calculation by Ford and Werner (FW) gives the exact multipole response of an isotropically conducting, nonmagnetic sphere to a uniform a.c. magnetic field in the presence of a d.c. magnetic field. ${ }^{3}$ There it was proposed that a measurement of the spectrum of longitudinal helicons, oscillations excited when the applied a.c. and d.c. fields are collinear, could be used to determine the magnetoresistance and Hall coefficient. Our experiments show that this calculation accurately predicts the response of potassium spheres in both absolute magnitude and phase.

A schematic diagram of the experimental apparatus is shown in Fig. 1. A uniform a.c. magnetic field (a few gauss) is applied to the sample using the a.c. drive solenoid, which is aligned coaxially with a superconducting solenoid. Inside the a.c. drive solenoid, a dummy coil, placed some distance from the sample and connected in series opposition is used to suppress the voltage induced directly in the detector coil by the uniform a.c. magnetic field. The signal induced in the detector coil by the sample is directly proportional to the time derivative of the induced magnetic dipole moment and is measured with a two-phase lock-in amplifier. ${ }^{4}$ The reference phase is determined by measuring with the signal channel the a.c. magnetic field at the sample site using the movable sensing coil. In FW the induced a.c. magnetic multipole moments are shown to depend on the two parameters $V=4 \pi \omega \sigma a^{2} / c^{i}$ 


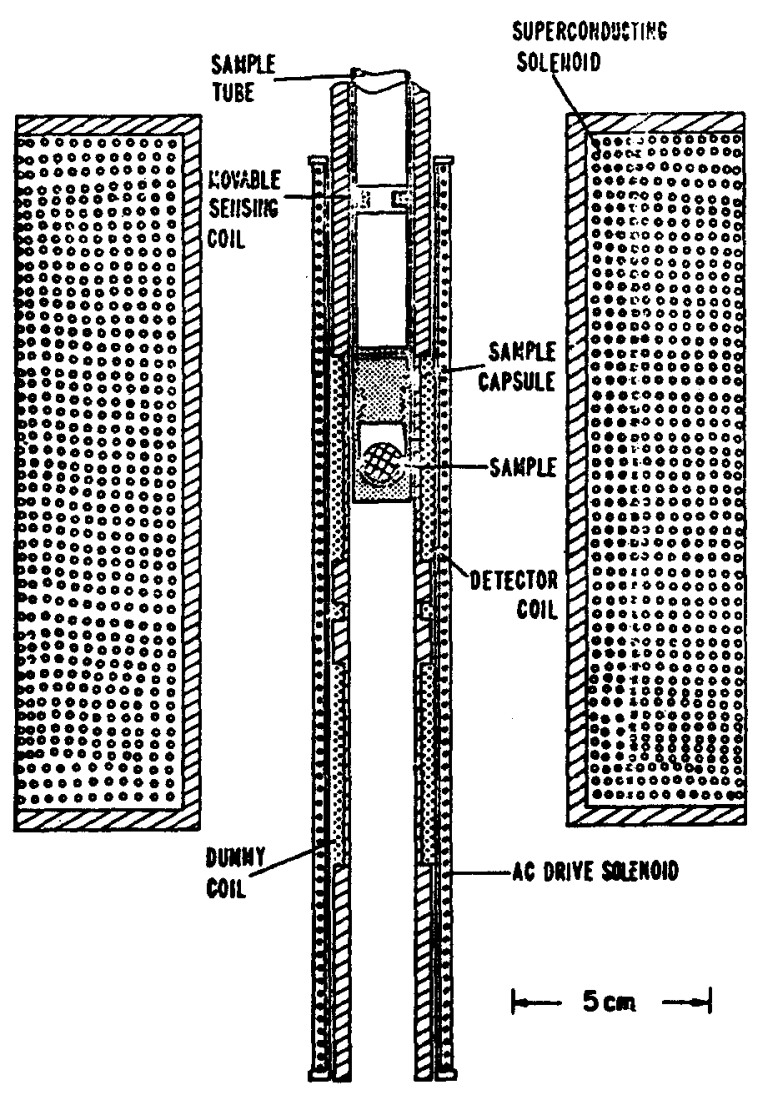

FIG. 1. Longitudinal plasma wave apparatus.

and $W=\omega_{c} \tau$. Here, $\omega$ is the angular frequency of the exciting field, $\sigma$ is the conductivity in the d.c. magnetic field $B_{0}, a$ is the radius of the sphere, $c$ the velocity of light, $\omega_{c}\left(=e B_{0} / m c\right)$ is the cyclotron frequency, and $\tau$ is the electron relaxation time. The fields due to moments higher than dipole can be large under certain conditions, but by using a long detector coil as shown in Fig. 1, sensitivity to the higher moments is largely eliminated.

With this apparatus we may easily determine the zero-field resistivity of a metal sphere at any temperature. For $B_{0}=0$, the a.c. magnetic dipole moment $M$ depends only on the parameter $V$, the amplitude of the a.c. field $B_{1}$ and the sphere radius $a$. The ratio $\operatorname{Re} M / \operatorname{Im} M$ is a monotonically increasing function of $V$ (see Fig. 1, in FW). This ratio is identically the ratio of the in-phase to the quadrature signals, and consequently allows an absolute determination of the resistivity independent of the coupling of the sample to the detector coil, provided the sphere radius is known.
Since potassium is a soft and very reactive metal, the accurate determination of the sample radius is a non-trivial problem. Fortunately, it is possible to measure the sample radius electrically since at high frequency $M$ becomes asymptotically $-B_{1} a^{3} / 2$, independent of the conductivity. An absolute measurement of $M$ thus provides an electrical determination of the sample radius. To make such a measurement, the coupling coefficient of the sample to the detector coil must be known. This coefficient is easily calculated, and we have also measured it by exciting with a known alternating current a small coil placed at the center of the detector coil. These calculated and measured results agree to better than $0.5 \%$. The accuracy of this radius determination technique was also confirmed to better than $0.5 \%$ on an aluminium sphere whose diameter could be conveniently measured by optical and mechanical means. Since there are no adjustable parameters here, this test may alternatively be viewed as a check upon the overall accuracy of the induced magnetic moment measurement. We thus have a simple in situ electromagnetic method for measuring the size of our potassium spheres.

The potassium spheres used in the present measurements were cast under outgassed, dry parafin oil in an accurately machined Kel-F mold, with solidification occurring under an excess pressure of He gas to insure that the substantial volume decrease of potassium upon freezing did not leave the mold partially empty. The starting material, obtained from MSA, ${ }^{6}$ was melted and outgassed in oil under vacuum at about $75^{\circ} \mathrm{C}$ for several hours prior to the casting operation. Microscopic examination of the samples showed them to be essentially perfect spheres with a smooth lustrous surface. Neutron diffraction photographic examination of one of the spheres showed it to be a homogeneous single crystal. Residual resistivity ratios at $4.2 \mathrm{~K}$ were typically 8000 with a further $30 \%$ decrease in the resistivity at $1.3 \mathrm{~K}$.

We show in Fig. 2 the longitudinal helicon spectrum obtained for a single crystal potassium sphere. The triangles and circles are experimental data and the dashed and solid lines give respectively $\operatorname{Re} M$ and $\operatorname{Im} M$ as calculated from the FW theory. Since the coupling coefficient is known, no adjustments of the ordinate have been made in this comparison between experiment and theory. This spectrum depends on the two parameters $V$ and $W$, or alternatively on the conductivity $\sigma\left(B_{0}\right)$ and the Hall coefficient $R_{H}\left(B_{0}\right)$. The resonance 


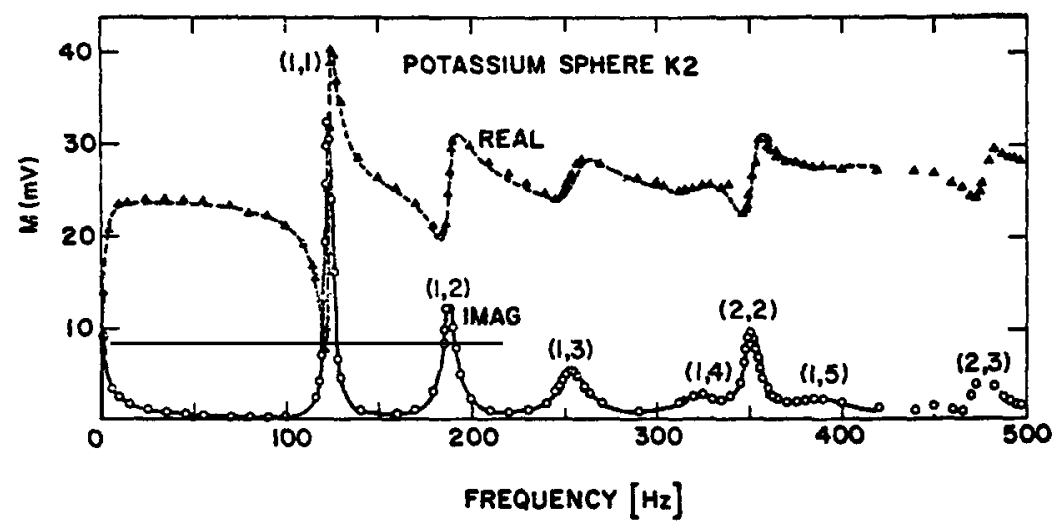

FIG. 2. The longitudinal helicon spectrum at $4.2 \mathrm{~K}$ in a field of $20.5 \mathrm{kG}$ for a single crystal potassium sphere $K 2$. Zero field $R R R=8200$. The electrically determined sphere diameter is $0.920 \mathrm{~cm}$. The parameters which result from fitting the theory to the data are $\omega_{c} \tau=95.30, \rho=1.006 \times 10^{-9} \Omega-\mathrm{cm}$. The zero field resistivity at $4.2 \mathrm{~K}$ is $0.876 \times 10^{-9} \Omega-\mathrm{cm}$. In order to convert the ordinate to magnetic moment in units of $B_{1} a^{3} / 2$, divide by 30.19 .

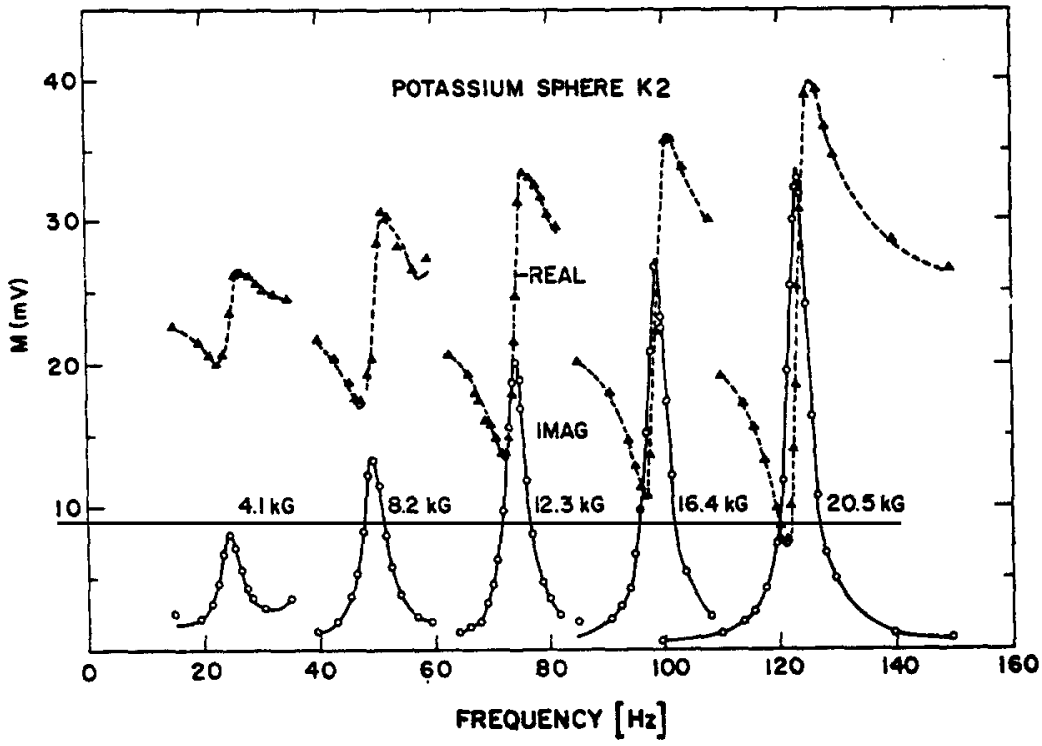

FIG. 3. Field dependence of the $(1,1)$ resonance for $K 2$ on the first cool-down. The solid and dashed lines are least square fits of the theory to the data. The parameters which lead to a fit are: at $4.1 \mathrm{kG}, \omega_{c} \tau=19.15, \rho=0.990 \times$ $10^{-9} \Omega-\mathrm{cm} ;$ at $8.2 \mathrm{kG} \omega_{c} \tau=38.29, \rho=1.002 \times 10^{-9} \Omega-\mathrm{cm} ;$ at $12.3 \mathrm{kG}, \omega_{c} \tau=57.7, \rho=0.998 \times 10^{-9} \Omega-\mathrm{cm}$; at $16.4 \mathrm{kG}, \omega_{c} \tau=76.6, \rho=1.08 \times 10^{-9} \Omega-\mathrm{cm}$ and at $20.5 \mathrm{kG}, \omega_{c} \tau=95.3, \rho=1.01 \times 10^{-9} \Omega-\mathrm{cm}$.

frequencies are determined primarily by $R_{H}$ and the width of the resonances primarily by $\sigma$.

It was shown in FW that the very low frequency behavior of $M(V, W)$ is independent of $W$ for the longitudinal geometry. At sufficiently low frequency we may therefore use the procedure discussed above for measuring the zero-field resistivity to determine the resistivity in the presence of an arbitrarily large d.c. magnetic field.

The field dependence of the $(1,1)$ resonance is displayed in Fig. 3. Fitting the theory to this data, by least squares analysis, produces values for the 


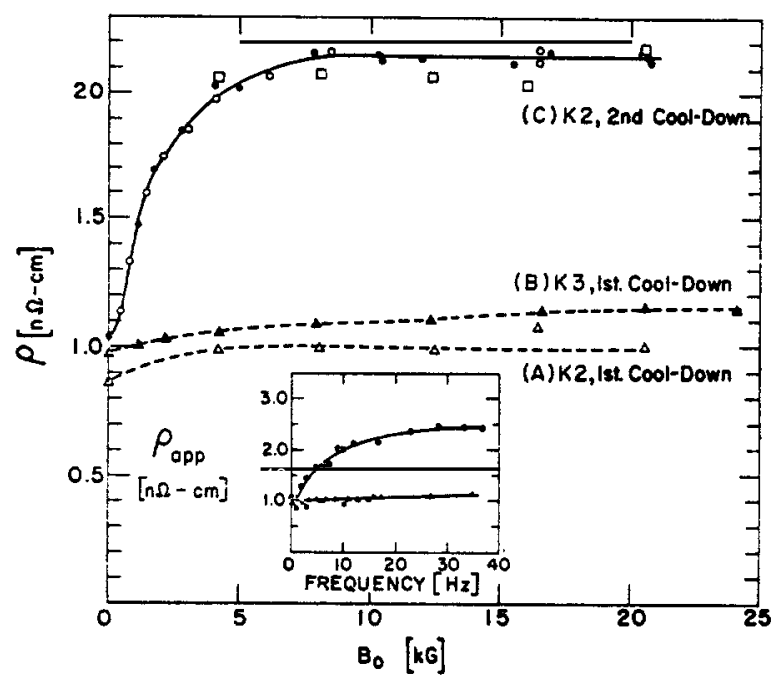

FIG. 4. magnetoresistance of two potassium spheres as measured by the low frequency method at $0.487 \mathrm{~Hz}$ and by the longitudinal helicon method. (A) $\rho\left(B_{0}\right)$ for $K 2$ using helicons, open triangles, (B) $\rho\left(B_{0}\right)$ for $K 3$ from the low frequency method, solid triangles, $(C)$ $\rho\left(B_{0}\right)$ for $K 2$ using the low frequency method on two different runs, open and closed circles; and using helicons, open squares. The insert shows the apparent frequency dependence of the resistivity for sample $K 2$ on the original cool-down and upon second cooling $\bullet$; and for sample $K 3$ on the original cool. down 4 .

field-dependent resistivity and the Hall coefficient. Magnetoresistance results obtained by this procedure, and by our low frequency measurements, are shown in Fig. 4. Measurements were made on sample $K 2$, after the original cool-down to $4.2 \mathrm{~K}$, and also after cycling to room temperature. It may be seen that the results for the magnetic field dependent resistivity obtained from the helicon data are reasonably close to the low frequency measurements. Data taken on the first cooling of both samples ( $K 2$ and $K 3$ ) shows a very weak magnetoresistance. At a field of $30 \mathrm{kG}, \omega_{c} \tau \approx 140$ for these samples. The zero-field resistivity of sample $K 2$, on its second cool-down shows a large apparent frequency dependence as displayed in the insert. As the exciting frequency increases, the present low frequency method more strongly emphasizes the currents close to the surface. A reasonable interpretation of this apparent frequency dependence of the resistivity is that impurities diffused into the sample from the surface while it was at room temperature, and thus increased the resistivity of the material close to the surface. It is interesting to note that the sample history which leads to a large apparent frequency dependence of the resistivity also leads to a large magnetoresistance. The implication that surface or near surface scattering is related to the magnetoresistance requires further examination, and conclusions drawn from the present data would be premature.

By measuring the frequency of the $(1,1)$ resonance we have found that the high field Hall coefficient is the same (to within $0.2 \%$ ) at $1.3 \mathrm{~K}$ and $4.2 \mathrm{~K}$, in agreement with the results of Chimenti and Maxfield. ${ }^{7}$ For fields above $4 \mathrm{kG}$ we measured the Hall coefficient for sample $K 2$ on the first and second cool-downs and found $R_{H}=(4.591 \pm 0.027) \times 10^{-10} \mathrm{~m}^{3} / \mathrm{C}$, and $(4.633 \pm 0.031) \times 10^{-10} \mathrm{~m}^{3} / \mathrm{C}$, respectively, independent of field. These results are respectively $2.8 \%$ and $3.8 \%$ higher than the free electron value. The high frequency helicon transmission measurements of Chimenti and Maxfield also gave a value of $R_{H}$ somewhat higher than the free electron value, whereas the d.c. measurements of Siebenmann and Babiskin gave $R_{H}$ in agreement with free electron theory. ${ }^{8}$

In this paper we have (a) presented the first observation of the longitudinal helicon spectrum in a metal, (b) demonstrated a new low frequency technique for the quantitative measurement of the resistivity in an arbitrarily large magnetic field, (c) presented data on the magnetoresistance of potassium showing a saturating behavior [high field Kohler slope $<7 \times 10^{-4}$ ], al though the change in the resistivity with magnetic field is sample dependent, and (d) found an apparent frequency dependence of the resistivity, which from the nature of the induced currents suggests that surface or near surface scattering may be an important aspect of the longstanding magnetoresistance problem in potassium metal.

Acknowledgements - We would like to acknowledge some very useful discussions with A.W. Overhauser on this work. The technical assistance of B. Poindexter has been extremely valuable. 


\title{
REFERENCES
}

1. These oscillations closely resemble the magnetic modes first observed by CARDONA M. and ROSENBLUM B., Phys. Rev. 129,991 (1963), in experiments on InSb spheres in the geometry in which the microwave magnetic field is collinear with the d.c. magnetic field.

2. See the review by DELANEY J.A. and PIPPARD A.B., Rep. Prog. Phys. 35, 677 (1972), and by MAXFIELD B.W., Am. J. Phys. 37, 241 (1969).

3. FORD G.W. and WERNER S.A., Phys. Rev. B 8, 3702 (1973).

4. Princeton Applied Research Corp., Model 129.

5. A similar method was used successfully by ZIMMERMAN J.E., Rev. Sci. Instr. 32, 402 (1960).

6. MSA Research Corp., Evans City, Pa. 10633, U.S.A.

7. CHIMENTI D.E. and MAXFIELD B.W., Phys. Rev. B 7, 3501 (1973).

8. SIEBENMANN P.G. and BABISKIN J., Phys. Rev. Lett. 30, 380 (1973).

\begin{abstract}
Wir haben in Kalium das Spektrum der longitudinalen Helikonoszillationen beobachte. Wir haben diese Oszillationen benutzt, um die magnetische Widerstandsveränderung und den Hall Koeffizienten zu messen. Zusätzlich wird eine Niederfrequenzmethode zur Messung der magnetischen Widerstandsveränderung anhand von Experimenten an einkristallinen Kaliumkugeln demonstriert. Die magnetische Widerstandsveränderung erreicht einen Sättigungswert der von der Probenbehandlung abhängt.
\end{abstract}

